\title{
In-vivo microscopy of the rat endometrial subepithelial capillary plexus during the oestrous cycle and after ovariectomy
}

\author{
P. A. W. Rogers and A. M. Macpherson \\ Department of Obstetrics and Gynaecology, Monash University, Monash Medical Centre, \\ 246 Clayton Road, Clayton, Victoria 3168, Australia
}

\begin{abstract}
Summary. Blood flow through the endometrium was visualized by using incident-light fluorescence miscroscopy and a video image recorded for later detailed analysis. The subepithelial microvascular density was calculated for each day of the oestrous cycle and at 7 days after ovariectomy. The results showed that the microvasculature was significantly more dense at dioestrus I, pro-oestrus, and after ovariectomy than at oestrus, with dioestrus II being in between. Mean capillary path lengths running from arteriole to venule were longest at pro-oestrus, followed by oestrus, dioestrus II, dioestrus I, and shortest after ovariectomy. The results suggest that endometrial growth and regression precede microvascular growth and regression. The technique of in-vivo microscopy provides an important new avenue for investigating the role of local factors in the control of the endometrial microcirculation.
\end{abstract}

Keywords: microcirculation; endometrium; rat; oestrous cycle; in-vivo microscopy

\section{Introduction}

The endometrial subepithelial capillary plexus continually changes in a cyclic fashion in response to oestrogen and progesterone during the reproductive life of the female. This is in contrast to most other vascular beds, which unless injured maintain a high degree of stability throughout adult life. In some primate species, including man, endometrial changes include significant new vessel growth or angiogenesis, followed by sloughing and regression during menstruation. In addition to these structural changes, there are also major changes in endometrial blood flow and vascular permeability in response to oestrogen and progesterone (Finn \& Porter, 1975). Although these vascular changes are brought about by circulating hormones, it is becoming increasingly clear that control of the endometrial microvasculature occurs primarily through local mechanisms (Sheppard, 1984). Understanding of these local mechanisms is far from complete, despite their relevance to such diverse issues as infertility, contraception and dysfunctional uterine bleeding.

Techniques developed to measure uterine blood flow in large species such as the sheep have shown that blood flow to the whole uterus is increased by oestrogen, and that this increase is reduced by progesterone and blocked by the protein synthesis inhibitor cycloheximide (Makowski, 1977). However, suitable techniques for studying endometrial microvascular function in vivo in small animals such as the rat have not been developed to date. Such methodology would provide valuable information towards resolving the current disagreement about changes in uterine blood flow during the rat oestrous cycle. Harvey \& Owen (1976), using radioactive microspheres to measure combined endometrial and myometrial blood flow, found highest flow per $100 \mathrm{~g}$ of uterine tissue at pro-oestrus followed by oestrus, with lowest rates at metoestrus and dioestrus I. By contrast, Kopin \& Wurtman (1963) using ${ }^{42} \mathrm{~K}$ in normal saline found that whole uterine blood flow per unit weight was lowest at oestrus, followed by metoestrus, then pro-oestrus, and highest at 
dioestrus. This observation was supported by the work of Einer-Jensen (1976), who injected ${ }^{85} \mathrm{Kr}$ in saline into the aorta and measured clearance of radioactivity from the uterine lumen with a Geiger-Muller probe. This author reported endometrial flow rates of $0.88 \pm 0.08 \mathrm{ml} / \mathrm{g} / \mathrm{min}$ at oestrus and $1.60 \pm 0.15 \mathrm{ml} / \mathrm{g} / \mathrm{min}$ at dioestrus. It is possible that these conflicting results are due to the different techniques employed, since microspheres may not enter the smaller microvessels and can thus underestimate blood flow. It is also relevant to note that only the study of Einer-Jensen (1976) provides data on endometrial blood flow alone; the studies of Harvey \& Owen (1976) and Kopin \& Wurtman (1963) combine myometrial and endometrial flow rates.

Clearly, to resolve this issue it would be of immense benefit to be able to visualize by in-vivo techniques the endometrial microvascular bed at each stage of the oestrous cycle. The aim of the present study was to develop such a technique for the rat endometrial subepithelial capillary plexus based on that described for gut by Bohlen \& Gore (1976), and to use this new methodology to report changes in endometrial microvascular density and appearance during the oestrous cycle and after ovariectomy.

\section{Materials and Methods}

Experimental animals. Sprague-Dawley rats (female, outbred, aged 3-6 months) used in this study were housed under standard conditions ( $14 \mathrm{~h}$ light, $10 \mathrm{~h}$ dark; lights on $06: 00 \mathrm{~h}$ ). Before the experiment vaginal smears were taken daily from each animal to confirm regular 4-day oestrous cycles (Rogers \& Gannon, 1981).

The technique for preparation and viewing of the endometrial subepithelial capillary plexus was as follows: rats were maintained under anaesthesia using Avertin, $30 \mathrm{mg} / 100 \mathrm{~g}$ body weight i.p. [stock solution: $5 \mathrm{~g} \mathrm{2,2,2-}$ tribromoethanol (Merck, Schuchardt, FRG) plus $5 \mathrm{ml}$ butan-2-ol (BDH Chemicals, Poole, Dorset, UK) working solution: $0.25 \mathrm{ml}$ stock plus $10 \mathrm{ml} 0.9 \%$ (w/v) sodium chloride], the right flank shaved, and a lateral abdominal incision made. All bleeding during surgery was minimized by cautery. Using careful handling the ovarian end of the uterine horn was exteriorized and held in place by haemostats attached to the fat body. A microcautery was then used to cut a small hole through the uterine wall close to the ovarian end of the uterus. Depending on the stage of the oestrous cycle (and hence the luminal diameter of the uterus) a glass rod of approximately equivalent diameter to the uterine lumen was then inserted into the uterus and pushed about $15-20 \mathrm{~mm}$ towards the cervical end. The microcautery was then used to start a cut along the ventral margin of the uterus, halfway between the mesometrial and antimesometrial borders, to the end of the glass rod. During this process the glass rod acted as a heat sink to prevent damage to the endometrial vasculature on the side of the lumen opposite to the cut. Further heat damage was minimized by dripping saline $(0.9 \%(\mathrm{w} / \mathrm{v}) \mathrm{NaCl})$ onto the cut immediately after the cutting process. Use of the microcautery was kept to the minimum sufficient to ensure that bleeding did not occur once the uterus was opened. The cut made in this way with the cautery did not extend through the thickness of the endometrium, but once blood flow along the line of the cut had been stopped by the actions of the cautery, a fine pair of scissors was used to finish the cut through to the lumen. From this point on, regular application of saline to the uterine endometrial surface prevented drying. Fine cotton thread stitched through the outermost edge of each end of the opened section of uterus was then used to position the uterus carefully over a specially designed Perspex microscope stage (see Fig. 1). The animal stage is based on the design of Bohlen \& Gore (1976), although the thermal block has been modified from a square to a trapezium when viewed from above to enable the rat to be moved as close as possible to the tissue pedestal, and hence minimize the uterine exteriorization necessary. The viewing window in the stainless-steel lid has been covered by a glass coverslip glued to the under side of the lid. Once the required portion of the endometrium had been positioned over the tissue pedestal the stainless-steel lid was carefully placed onto the stage and pressed down until the endometrial surface made good contact with the glass coverslip. Care was taken to ensure that the stainless-steel lid did not compromise blood flow due to excessive downwards pressure on the tissue.

Video visualization of endometrial microvasculature. Once set up, the endometrial preparation was left for $30 \mathrm{~min}$ to allow equilibration before starting experimental observations. At $5 \mathrm{~min}$ before starting observations, each rat was injected with $10 \mathrm{mg}$ FITC-Dextran (mol. wt 150000 ; Sigma, St Louis, MO, USA) a fluorescent marker which emits strongly between the wavelengths of $500-540 \mathrm{~nm}$, in $0.2 \mathrm{ml}$ saline via the tail vein. The whole Perspex stage was then fixed onto the stage drive of a Zeiss (Oberkochen, West Germany) ACM incident-light fluorescence microscope for viewing. The objective used for this work was a Leitz (Heerbrugg, Switzerland) $\times 25$ long working distance, sea-water immersion lens. Video images were taken with a high-sensitivity, video SIT (single intensified silicon target image tube) camera (COHU, San Diego, CA, USA), and recorded on a video cassette recorder with stop frame capabilities (Sony B SL-HF950, Tokyo, Japan).

Using this microscopy technique it was possible to focus onto and visualize vasculature that ran deep within the endometrium. For the purposes of this study, video recordings were always made of the most superficial vessels at each site (i.e. those just under the epithelium). Vessels below the most superficial plane of focus were therefore not recorded. 
(a)

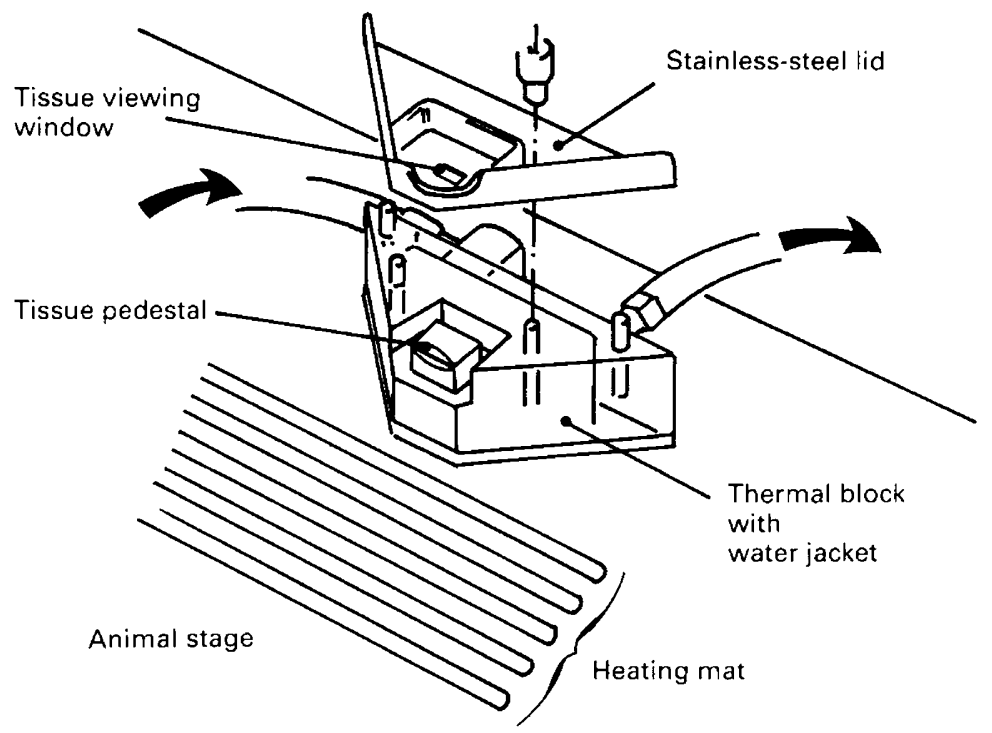

(b)

Mucosal surface of uterus

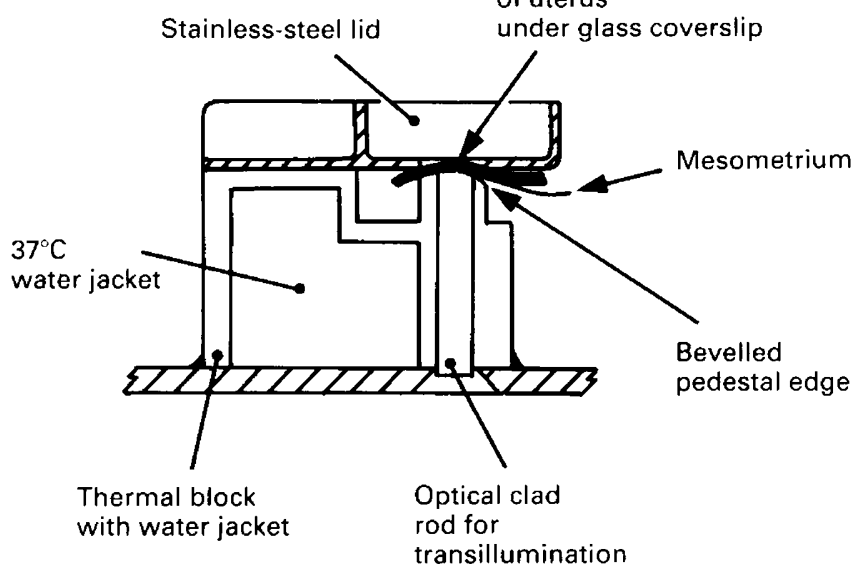

Fig. 1. Diagram of the specially designed Perspex microscope stage used for in-vivo microscopy of the rat endometrium. Dimensions of thermal block: $18 \mathrm{~mm}$ high by $15 \mathrm{~mm}$ along the shortest edge by $70 \mathrm{~mm}$ along the longest edge. Dimensions of tissue pedestal: $5 \mathrm{~mm}$ high by $10 \mathrm{~mm}$ by $6 \mathrm{~mm}$.

Areas of endometrial subepithelial capillary plexus for study were selected from as close to the anti-mesometrial border as possible, although at times identification of this area was not always certain. In general terms the area selected for viewing was always the one providing the best image, with the best blood flow and the most patent vessels. These selection criteria were similar for each animal. Areas of the endometrium showing signs of thermal or surgical injury (i.e. haemorrhage or higher than normal levels of stasis) or which had become abnormally permeable (as assessed by excessive leakage of FITC-Dextran from the vessels into the tissues) were excluded from this study. 
In some uteri epithelial folds or channels were observed running across the surface of the endometrium. When this occurs the endometrial subepithelial capillary plexus drops out of the superficial plane of focus to run deeper within the endometrium. To ensure comparable results from animal to animal, areas of epithelial folding were also excluded from this study.

Once the 30 -min equilibration period was completed a 30 -min observation period was begun. During this time a video recording was made of as large an area of microvasculature of normal appearance as possible. A montage was then constructed using a video-graphic printer (UP-811 Sony) from which measurements of vascular density were made.

Experimental design. The 25 rats used were studied on the mornings of the 4 days of the oestrous cycle (5 on each day), and on the morning of the 7 th day after ovariectomy $(\mathrm{N}=5)$. All observations were made between 10:30 and $12: 30$ h over the course of the experimental period.

Analysis of video montages of the subepithelial capillary plexus. Each montage was scored for microvascular density by tracing vessels from an area $295 \mathrm{~mm} \times 210 \mathrm{~mm}$ onto an acetate sheet, overlaying this onto a grid with $20 \times 20 \mathrm{~mm}$ squares and counting the number of times a vessel intersected a grid line. The $295 \times 210 \mathrm{~mm}$ area measured in this way corresponded to an area of endometrium $1009 \times 718 \mu \mathrm{m}$ at original magnification. To correct for individual subjectivity both in selecting areas of montage to trace and in tracing the vessels onto the acetate sheet, 3 independent observers repeated the microvascular tracing and density scoring.

One representative montage was selected from each experimental group for further detailed analysis by one observer. For each of these, blood flow direction in every patent vessel was recorded from multiple re-runs of the video tape. Once blood flow directions were known it was possible to identify points at which arterioles entered into the subepithelial capillary plexus and places where venules left. Capillary lengths running within the subepithelial plexus between the feeding arteriole and the draining venule were then measured with a map measuring-wheel and a mean path length was calculated for each montage.

\section{Results}

Relative endometrial microvascular densities calculated by 3 independent observers are given in Table 1 . The analysis of the data was by analysis of variance. The experimental variance of each of the observers was estimated, and using Bartlett's test it was found that the variances were equal. There was no suggestion of any interaction between the observer effects and the rat group effects. Comparisons of sample means demonstrate that the microvascular density at oestrus was significantly reduced compared with dioestrus I, pro-oestrus, and ovariectomy $(P<0.05)$, but not dioestrus II. Significant differences in endometrial microvascular density were not found between any other stages of the cycle.

Mean capillary path lengths for the 5 montages analysed in detail are given in Table 2 . These figures correspond to a degree with the data in Table 1, with the shortest path lengths and the most dense microvasculature both being at dioestrus I or after ovariectomy. However, the longest capillary path lengths occurred at pro-oestrus whilst the microvascular density was lowest at oestrus.

Overall, the endometrial subepithelial capillary plexus maintained a similar topology through the oestrous cycle and after ovariectomy. Each arteriole entering the capillary plexus usually drained via one or two venules, although up to 5 or 6 venules draining one arteriole were sometimes seen. Occasionally two arterioles fed the same venule, although this was less common. The whole capillary network was very anastomotic, resulting in many vessel segments with 'trapped' plasma and blood cells. Blood flow reversals were common in these interconnecting capillaries, even at times of the oestrous cycle when flow reversals were not seen in larger vessels. Blind-ending capillary buds or 'spikes' were present at all stages of the cycle, although it was not possible to discern whether these represented growing, steady state, or regressing vessels.

In addition to objective microvascular density measurements, a number of semi- or nonquantitative observations were also recorded at each stage of the cycle. These included information on myometrial activity within the microscope preparation, blood flow rates, leakage of FITCDextran from the microvessels into the tissues, apparent signs of angiogenesis or regression, 
Table 1. Relative microvascular densities for the endometrial subepithelial capillary plexus in 5 groups of 5 rats at different stages of the oestrous cycle and after ovariectomy

\begin{tabular}{|c|c|c|c|c|c|}
\hline \multirow{2}{*}{$\begin{array}{l}\text { Rat } \\
\text { no. }\end{array}$} & \multirow{2}{*}{$\begin{array}{l}\text { Cycle } \\
\text { stage }\end{array}$} & \multicolumn{3}{|c|}{$\begin{array}{l}\text { Microvascular density, } \\
\text { relative scores }\end{array}$} & \multirow{2}{*}{$\begin{array}{l}\text { Mean } \pm \text { s.d. } \\
\quad(n=15)\end{array}$} \\
\hline & & 1 & 2 & 3 & \\
\hline 1 & Oestrus & 266 & 267 & 240 & \multirow{5}{*}{$212 \cdot 2 \pm 29.9$} \\
\hline 2 & Oestrus & 219 & 212 & 205 & \\
\hline 3 & Oestrus & 229 & 219 & 216 & \\
\hline 4 & Oestrus & 194 & 159 & 184 & \\
\hline 5 & Oestrus & 190 & 189 & 192 & \\
\hline 6 & Dioestrus I & 294 & 289 & 304 & \multirow{5}{*}{$274 \cdot 3 \pm 27 \cdot 8$} \\
\hline 7 & Dioestrus I & 326 & 282 & 305 & \\
\hline 8 & Dioestrus I & 291 & 263 & 246 & \\
\hline 9 & Dioestrus I & 274 & 245 & 273 & \\
\hline 10 & Dioestrus I & 227 & 247 & 249 & \\
\hline 11 & Dioestrus II & 269 & 220 & 221 & \multirow{6}{*}{$241 \cdot 9 \pm 30 \cdot 6$} \\
\hline 12 & Dioestrus II & 276 & 243 & 275 & \\
\hline 13 & Dioestrus II & 239 & 173 & 197 & \\
\hline 14 & Dioestrus II & 288 & 245 & 243 & \\
\hline 15 & Dioestrus II & 258 & 237 & $244^{\prime}$ & \\
\hline 16 & Pro-oestrus & 220 & 203 & 194 & \\
\hline 17 & Pro-oestrus & 232 & 253 & 247 & \multirow{4}{*}{$267 \cdot 4 \pm 46 \cdot 0$} \\
\hline 18 & Pro-oestrus & 299 & 346 & 290 & \\
\hline 19 & Pro-oestrus & 319 & 310 & 326 & \\
\hline 20 & Pro-oestrus & 256 & 262 & $254^{\prime}$ & \\
\hline 21 & Ovariectomy & 305 & 272 & 280 & \multirow{5}{*}{$280.6 \pm 48.6$} \\
\hline 22 & Ovariectomy & 293 & 295 & 290 & \\
\hline 23 & Ovariectomy & 257 & 280 & 251 & \\
\hline 24 & Ovariectomy & 221 & 178 & 239 & \\
\hline 25 & Ovariectomy & 365 & 349 & 334 & \\
\hline
\end{tabular}

Bartlett's test showed that the theoretical variances of the observers were equal. Analysis of variance showed that the mean of the oestrus group is significantly lower than the means of all the other groups $(P<0.05)$ except that of the dioestrus II group.

Table 2. Mean endometrial subepithelial capillary path lengths for a single rat from each experimental group

\begin{tabular}{|c|c|c|c|}
\hline $\begin{array}{l}\text { Rat } \\
\text { no. }\end{array}$ & $\begin{array}{l}\text { Cycle } \\
\text { stage }\end{array}$ & $\begin{array}{l}\text { Mean } \pm \text { s.d. } \\
\text { capillary } \\
\text { path length } \\
(\mu \mathrm{m})\end{array}$ & $\begin{array}{c}\text { No. of } \\
\text { observations } \\
\text { (one observer) }\end{array}$ \\
\hline 1 & Oestrus & $322 \pm 91$ & 32 \\
\hline 9 & Dioestrus I & $212 \pm 102$ & 32 \\
\hline 15 & Dioestrus II & $228 \pm 96$ & 33 \\
\hline 20 & Pro-oestrus & $345 \pm 91$ & 33 \\
\hline 25 & Ovariectomy & $195 \pm 68$ & 33 \\
\hline
\end{tabular}

overall ease of video-imaging. These observations form the basis of the following brief description of the endometrial microvasculature at each stage of the cycle:

Oestrus. Capillaries appeared narrow (Fig. 2a) and blood flow was often sluggish with intermittent stoppages and/or flow reversals. Haematocrit within and between vessels was varied with 

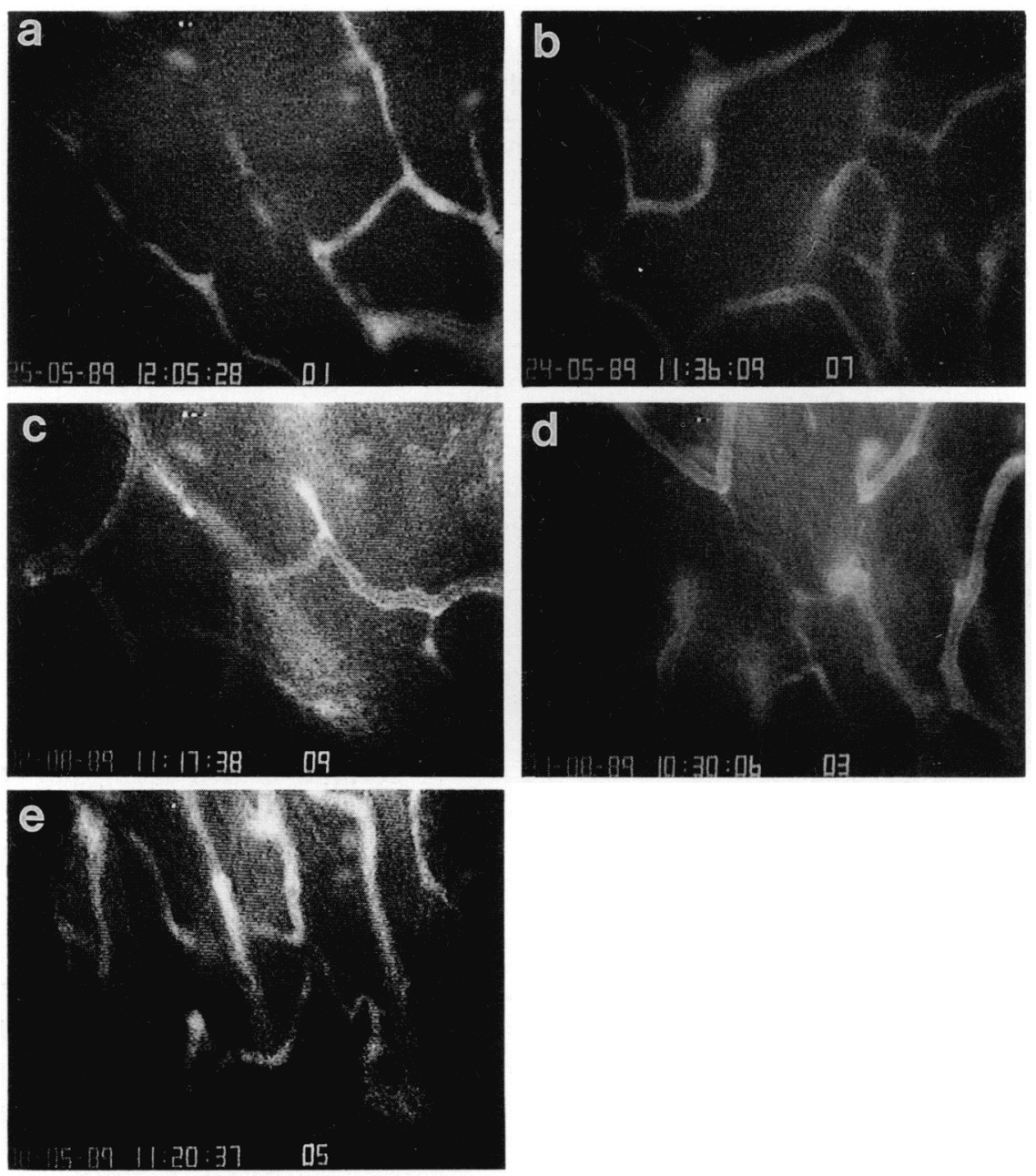

Fig. 2. In-vivo fluorescence microscopy of the rat endometrial subepithelial capillary plexus following $10 \mathrm{mg}$ FITC - Dextran (mol. wt 150000 ) i.v. $\times 250$. Photographs are taken from a video recording on the morning of: (a) oestrus, note the low density of blood vessels compared to other stages, and the narrow capillaries; (b) dioestrus I, note the short distance between vessel junctions; (c) dioestrus II, note the apparently blind ending microvascular buds; (d) prooestrus, note the presence of very thin new vessels and capillary buds, and dilated microvessels; (e) 7 days after ovariectomy.

areas of clear plasma trapped between large plugs of red blood cells. Myometrial movements were frequent and strong in amplitude. FITC-Dextran leakage was not a significant problem over the 30-min observation period. Overall, the size of the uterus at oestrus made surgery and video imaging relatively easy.

Dioestrus $\boldsymbol{I}$. Blood flow in patent vessels was rapid and constant with few stoppages or reversals. However, up to $30 \%$ of vessels showed stasis and contained just plasma without any blood cells. Myometrial movements were slightly less frequent and of lower amplitude than at oestrus. The microvascular bed was densely packed with shorter distances between vessel junctions than at oestrus (Fig. 2b). The increased density of the vascular bed made imaging more 
of a problem due to fluorescence from underlying vessels. FITC-Dextran leakage was not a problem.

Dioestrus II. Blood flow was generally rapid and constant with few stoppages or reversals except in interconnecting capillaries. Myometrial activity was almost continuous with slow movements of gentle to moderate amplitude. In 1 or 2 preparations, leakage of FITC-Dextran was significant, although the remainder appeared unaffected. Numerous microvascular buds were apparent (Fig. 2c).

Pro-oestrus. Blood flow was good with few stoppages or reversals. Myometrial activity was almost non-existent. Several very thin new vessels and capillary buds were seen between the main microvessels (Fig. 2d), which appeared more dilated that at other stages of the cycle. FITCDextran leakage was not apparent. Pro-oestrus was the best stage of the cycle for video-imaging from a technical viewpoint due to the large size of the uterus and the minimal myometrial movements.

After ovariectomy. Blood flow was good with few stopages or reversals. Almost no myometrial movement was observed. By 7 days after ovariectomy the uterus had regressed in size considerably, making surgery and handling quite difficult. FITC-Dextran leakage was not a problem. Capillaries did not appear as dilated as at pro-oestrus despite good flow (Fig. 2e).

\section{Discussion}

The results from this study provide for the first time an accurate in-vivo description of the rat endometrial subepithelial capillary plexus and show significant changes in network density during the oestrous cycle and after ovariectomy. It seems probable that the changing vascular densities during the cycle are caused by alterations in endometrial tissue volume and configuration, rather than by vessel growth and regression. At oestrus, for example, the significantly reduced microvascular density was primarily due to the rapid increase in endometrial volume (measured as an increase in uterine wet weight: see Kopin \& Wurtman, 1963; Harvey \& Owen, 1976). This increase in endometrial volume presumably results from an increase in microvascular permeability causing a nett fluid movement from the vasculature into the tissues. Such an increase in tissue fluid, or oedema, will raise the hydrostatic pressure within the endometrium, thus tending to compress the vasculature, and, assuming vascular perfusion pressure does not alter, will reduce blood flow to the superficial layers of the endometrium. That this happens is supported by the results of Einer-Jensen (1976), who found endometrial blood flow rates of $0.88 \mathrm{ml} / \mathrm{g} / \mathrm{min}$ at oestrus and $1.60 \mathrm{ml} / \mathrm{g} / \mathrm{min}$ at dioestrus in rats by using inert gas clearance techniques. By the morning of dioestrus I uterine wet weight has decreased significantly, moving the blood vessels closer together as oedema reduces. At dioestrus II changes associated with increasing concentrations of circulating oestrogen (Butcher et al., 1974; Nequin et al., 1979) may be having an effect in some animals. Uterine wet weight may be increasing slightly, and hence vascular density decreasing, compared with the day before. The most striking changes in endometrial structural organization occur at pro-oestrus. At this time cervical constriction results in the massive accumulation of luminal fluid, distending the uterus to many times its diameter at dioestrus (Kennedy \& Armstrong, 1975). At this time the endometrium becomes stretched and thin. This stretching has 2 effects on the endometrial vasculature. Firstly, the superficial microvessels will be elongated and pulled further apart. The second effect, however, will be to pull the deeper vessels up into the same plane of focus as the surface vessels. Therefore, although the capillary length between each arteriole and venule is greatly increased at pro-oestrus (see Table 2), the vascular density does not decrease as dramaticaly as at oestrus (see Table 1).

The improved understanding of configurational changes in the endometrial capillary plexus resulting from this study may provide new insight into the local mechanisms regulating microvascular growth and regression. During pro-oestrus the microvasculature is under considerable 
force, stretching and elongating individual vessels. This stretching may provide the stimulus necessary to induce endothelial cell mitosis and hence new vessel growth. At oestrus the luminal fluid is lost, although the endometrial oedema present at this time continues to provide a strong force separating and elongating the microvasculature. Unlike the force generated by the luminal fluid at pro-oestrus (which is primarily unidirectional and pushes the endometrium outwards), the oedema at oestrus is within the endometrium, thus providing a force which greatly expands the tissue volume. As a result microvascular density is significantly reduced, as is blood perfusion per unit mass of tissue (Einer-Jensen, 1976). These changes at oestrus will result in some cells within the endometrium no longer being within the normal functional distance of the vasculature; a situation that will presumably stimulate angiogenesis.

During dioestrus the endometrial tissue volume decreases, the lumen remains shut, and microvascular density increases while capillary length decreases. Under these conditions the endometrium is in a sense 'over-serviced' with blood vessels and can afford some to become redundant. This may well be reflected by the increased number of anastomosing capillaries showing stasis at this time. By 7 days after ovariectomy the endometrial stroma has regressed dramatically. In contrast, microvascular density is at its greatest and capillary path length its shortest. These results further support the view that the endometrial microvasculature is responding to and following the changes in tissue regression rather than causing them. In summary, it is possible that the endometrium initially generates the stimuli for angiogenesis and vascular regression by controlling the nett movement of fluid in and out of the microvasculature. The physical stimuli provided by these rapid changes in fluid balance may then be replaced by changes in tissue volume due to stromal cell growth or death at a later stage.

The endometrial microvascular changes reported in this study provide strong morphological evidence to support the physiological observations of Mitchell \& Yochim (1968a, b). These authors report an average intrauterine oxygen tension $\left(\mathrm{PO}_{2}\right)$ of $25.7 \mathrm{mmHg}$ at oestrus, rising to $47.6 \mathrm{~mm} \mathrm{Hg}$ at dioestrus I, with other stages of the oestrous cycle being between these two values. Furthermore, over 5 days after ovariectomy (performed on the day of oestrus), intrauterine $\mathrm{PO}_{2}$ rose from $25 \cdot 7$ to $61.4 \mathrm{mmHg}$. Although blood perfusion rates and tissue respiration (which were not measured in the present study) will clearly play major roles along with microvascular density in determining $\mathrm{PO}_{2}$ values, the strong correlation between increasing $\mathrm{PO}_{2}$ and increasing microvascular density suggests a strong link between structure and function.

The level of myometrial activity reported in this study for each day of the oestrous cycle is in general agreement with the observations of Ishikawa \& Fuchs (1978). This result provides evidence that the surgery required to prepare the uterus for in-vivo microscopy had not unduly interrupted normal myometrial function.

The technique of in-vivo microscopic visualization of the endometrial subepithelial capillary plexus allowed collection of previously unobtainable information. Repeated or continuous observations over a period of minutes to hours are now possible and the reactivity of single microvessels to different stimuli can be studied, as can differences between arterioles, capillaries and venules. Values such as blood flow direction, velocity, and vessel diameter can be measured with the appropriate video image-analysis equipment, and fluorescent tracers of different molecular weight can be used to follow changes in vascular permeability. The present technique also provides a more complete picture of the entire vascular space than do other methods, such as vascular casting in which anastomotic vessels will often fail to fill with casting medium (Rogers \& Gannon, 1981). Overall, despite the normal caveats associated with in-vivo observations following major surgery, this technique holds great promise in providing a better understanding of the local mechanisms controlling endometrial microvascular function.

This study was supported in part by a grant from NH\&MRC. We thank Dr Philip I. McCloud and Patricia F. Young from the Monash University Statistical Consulting Service for the statistical analysis of the results in Table 1; and Anne Grendon for typing the manuscript. 


\section{References}

Bohlen, H.G. \& Gore, R.W. (1976) Preparation of rat intestinal muscle and mucosa for quantitative microcirculatory studies. Microvasc. Res. 11, 103-110.

Butcher, R.L., Collins, W.E. \& Fugo, N.W. (1974) Plasma concentration of $\mathrm{LH}, \mathrm{FSH}$, prolactin, progesterone and estradiol-17 $\beta$ throughout the 4-day estrous cycle of the rat. Endocrinology 94, 1704-1708.

Einer-Jensen, N. (1976) Endometrial blood flow in rats. Hormone Res. 7, 49-55.

Finn, C.A. \& Porter, D.G. (1975) The action of ovarian hormones on the endometrium. In The Uterus, pp. 42-56. Ed. C. A. Finn. Elek Science, London.

Harvey, C.A. \& Owen, D.A.A. (1976) Changes in uterine and ovarian blood flow during the oestrous cycle in rats. $J$ Endocr. 71, 367-369.

Ishikawa, M. \& Fuchs, A.R. (1978) Electrical and mechanical activity of rat uterus in vivo during the estrous cycle. Am. J. Obstet. Gynaecol. 132, 611-619.

Kennedy, T.G. \& Armstrong, D.T. (1975) Loss of uterine luminal fluid in the rat: relative importance of changing peripheral levels of estrogen and progesterone. Endocrinology 97, 1379-1385.

Kopin, I.J. \& Wurtman, R.J. (1963) Flow of uterine blood and the oestrous cycle. Nature, Lond. 199, $386-387$.
Makowski, E.L. (1977) Vascular physiology. In Biology of the Uterus, pp. 77-99. Ed. R. M. Wynn. Plenum Press, New York.

Mitchell, J.A. \& Yochim, J.M. (1968a) Measurement of intrauterine oxygen tension in the rat and its regulation by ovarian steroid hormones. Endocrinology 83, 691-700.

Mitchell, J.A. \& Yochim, J.M. (1968b) Intrauterine oxygen tension during the estrous cycle in the rat: its relation to uterine respiration and vascular activity. Endocrinology 83, 701-705.

Nequin, L.G., Alvarez, J. \& Schwartz, N.B. (1979) Measurements of serum steroid and gonadotropin levels and uterine and ovarian variables throughout 4 day and 5 day estrous cycles in the rat. Biol. Reprod. 20, 659-670.

Rogers, P.A.W. \& Gannon, B.J. (1981) The vascular and microvascular anatomy of the rat uterus during the oestrous cycle. Aust. J. exp. Biol. Med. Sci. 59, 667-679.

Sheppard, B.L. (1984) The pathology of dysfunctional uterine bleeding. Clinics Obstet. Gynaecol. 11, 227-236.

Received 27 November 1989 